\title{
Digital medicine systems: an evergreening strategy or an advance in medication management?
}

\section{Alexander C Egilman, ${ }^{1,2}$ Joseph S Ross ${ }^{1,2}$}

10.1136/bmjebm-2019-111265

${ }^{1}$ Internal Medicine, Yale School of Medicine, New Haven, Connecticut, USA ${ }^{2}$ Center for Outcomes Research and Evaluation, Yale-New Haven Hospital, New Haven, Connecticut, USA

Correspondence to: Dr Joseph S Ross, Internal Medicine, Yale School of Medicine, New Haven, CT 06510,USA; joseph.ross@ yale.edu

\section{SLinked}

- http://dx.doi.org/10.1136/ bmjebm-2019-111204

\section{Check for updates}

( $)$ Author(s) (or their employer(s)) 2019. No commercial re-use. See rights and permissions. Published by BMJ.

To cite: Egilman AC, Ross JS. BMJ EvidenceBased Medicine 2019;24:203-204.
Poor adherence to prescribed medications is a major public health problem in the USA and worldwide, leading to substandard management of diseases and their associated symptoms, as well as increasing healthcare costs. ${ }^{1}$ The development of innovative technologies to improve medication adherence is important and may hold promise, but any new technologies need to be supported by robust evidence of patient benefit and safety prior to regulatory approval, clinical uptake and payer reimbursement.

Most technologies developed to improve medication adherence are ancillary or supportive: automated pill dispensers, mobile phone applications that remind patients to take their pills, or smart pill bottles that track when a pill is removed. ${ }^{2}$ Proteus Digital Health's ingestible sensor reimagines the way technology is used in medicine. The sensor is embedded within an oral tablet and transmits a signal to a wearable patch after exposure to stomach acid, providing patients and their clinicians with trackable ingestion data on medication use. The intent of the product is to improve medication adherence, but it may also have additional benefits, such as mitigating the risks of overdose and abuse. ${ }^{3}$ Since the sensor was cleared by the Food and Drug Administration (FDA) as a standalone device through the 510(k)-premarket notification process in 2012, Proteus has collaborated with several manufacturers to embed the sensor directly into FDA-approved drugs. ${ }^{4}$ In November 2017, Abilify MyCite, which combines the atypical antipsychotic, aripiprazole, with Proteus' digital sensor, became the furst FDA-approved digital medicine system, a drug-device combination that measures and reports medication ingestion. ${ }^{5}$

In this issue of BMJ Evidence-Based Medicine, Cosgrove et al systematically reviewed the evidence supporting FDA's approval of the precedent setting Abilify MyCite, as well as any additional evidence supporting use of the drug-device combination product. ${ }^{6}$ The authors conclude that regulatory approval was based on weak evidence, with no clinical studies demonstrating that Abilify MyCite was associated with better, or even noninferior, medication adherence than the older, readily available, Abilify tablet.

In total, the authors identified three clinical studies supporting FDA's approval, all non-comparative uncontrolled cohorts, which primarily examined the usability of the product among patients with disease, demonstrating that Abilify MyCite successfully tracks ingestion of aripiprazole. However, lacking comparator arms, these studies cannot provide evidence that Abilify
MyCite improved medication adherence, nor evidence of its effect on quality of life, psychiatric symptoms or remission. Additionally, the three trials provide limited safety data, particularly considering that Abilify MyCite is intended for chronic use. The longest trial was 16 weeks in duration and all the trials in aggregate included fewer than 200 participants, with no comparative data on gastrointestinal, skin or other adverse events.

The authors then reviewed the scientific literature, including all papers citing these trials, as well as news reports, finding that both the published literature and media conveyed an impression that Abilify MyCite was associated with important benefits, even though no such evidence had been demonstrated. Over half failed to acknowledge the lack of efficacy data from clinical trials and threefourths claimed benefits that were unsupported by the evidence.

These findings raise several concerns. First, FDA approval of Abilify MyCite without comparative efficacy data raises the concern that digital medicine systems may represent a new strategy to 'evergreen' drugs approaching the end of their market exclusivity periods. Evergreening involves a range of practices pharmaceutical manufacturers pursue to extend monopoly protection, most often by making minor changes to the dosing or formulation of older, readily available drugs, the newer versions of which offer marginal or no additional benefit to patients. ${ }^{7}$ These slightly modified drugs are often priced expensively relative to generic versions of the older drug. For example, a month's supply of Abilify MyCite in the US costs approximately 85 times that of generic aripiprazole. Because of these higher prescription drug costs, evergreening may also worsen patient outcomes, as the inability to afford drugs has been shown to be a major cause of non-adherence. ${ }^{8}$ Thus, any potential gains in adherence from digital medicine systems may be offset by their increased costs.

Additionally, digital medicine systems present unique ethical and safety concerns. For example, Cosgrove et $a l^{6}$ and other experts have raised concerns about the potential negative effects of giving ingestible drugs with sensors to patients with serious psychiatric illnesses, including some of whom may suffer from paranoia and delusions. ${ }^{9}$ Finally, further evaluation of the accuracy medication ingestion tracking is needed. One FDA reviewer of the Abilify MyCite New Drug Application noted that one study of healthy adults suggested the digital medicine system had a roughly $20 \%$ false negative rate, ${ }^{10}$ which could 
lead to an increased risk of overuse and overdose. Despite such concerns, limited preapproval safety data were collected, and FDA did not require any postmarketing studies at the time of approval that could generate reliable safety, or efficacy, data.

Several steps are needed to promote more rigorous evaluation of digital medicine systems to ensure that older drug products being brought to market as part of innovative delivery systems are improving outcomes for patients and not simply increasing profits for manufacturers through another evergreening strategy. First, the FDA should raise the evidentiary standards required for these products, ideally requiring clinical evidence of superiority as compared with the older drug products. In addition to examining patient adherence to medications, studies should measure additional patient-important outcomes such as quality of life, symptom burden and functional status. At a minimum, studies should use a non-inferiority design, as placebo control studies in this context would be unethical.

Second, clinicians should defer prescribing these products and payers should not provide coverage until rigorous evidence is generated demonstrating that digital medicine systems improve outcomes for patients and are worth the added costs. Finally, the FDA and the US government should consider measures to better incentivize manufacturers to develop and market generic versions of the non-digital products, even after market entry of the digital medicine system. Continued availability of the generic, non-digital versions will help prevent 'forced switches' that often accompany evergreening strategies, ${ }^{11}{ }^{12}$ and help ensure patient access to more affordable therapies, which may be a better option, particularly for those at lower risk for medication non-adherence.

Digital medicine systems have the potential to provide several benefits to patient care, including increasing medication adherence, providing real-time tracking of drug use and mitigating risks of overdose and abuse. ${ }^{13}$ However, until robust evidence of patient benefit and safety is generated, clinicians ought to be hesitant to prescribe Abilify MyCite and any future FDA-approved digital medicine systems.

Contributors ACE and JSR both contributed to the authorship of this editorial.

Funding This commentary was written as part of the Collaboration for Research Integrity and Transparency (CRIT) at Yale, funded by the Laura and John Arnold Foundation, which supports ACE and JSR.

Disclaimer The Laura and John Arnold Foundation played no role in the design and conduct of the study; collection, management, analysis and interpretation of the data; preparation, review or approval of the manuscript; and decision to submit the manuscript for publication.

Competing interests All authors have completed the ICMJE uniform disclosure form at www.icmje.org/coi_disclosure.pdf and declare: In the past 36 months, JSR has received research support through Yale University from Johnson \& Johnson to develop methods of clinical trial data sharing, from Medtronic and the Food and Drug Administration (FDA) to develop methods for postmarket surveillance of medical devices (U01FD004585), from the Food and Drug Administration to establish YaleMayo Clinic Center for Excellence in Regulatory Science and Innovation (CERSI) program (U01FD005938), from the Blue Cross Blue Shield Association to better understand medical technology evaluation, from the Centers of Medicare and Medicaid Services (CMS) to develop and maintain performance measures that are used for public reporting (HHSM-500-2013-13018I), from the Agency for Healthcare Research and Quality (R01HS022882), from the National Heart, Lung and Blood Institute of the National Institutes of Health (NIH) (R01HS025164), and from the Laura and John Arnold Foundation to establish the Good Pharma Scorecard at Bioethics International. ACE has no additional potential competing interests to disclose.

Patient consent for publication Not required.

Provenance and peer review Not commissioned; internally peer reviewed.

\section{References}

1. Osterberg L, Blaschke T. Adherence to medication. $N$ Engl J Med 2005;353:487-97.

2. Granger BB, Bosworth HB. Medication adherence: emerging use of technology. Curr Opin Cardiol 2011;26:279-87.

3. Chai PR, Carreiro S, Innes BJ, et al. Oxycodone ingestion patterns in acute fracture pain with digital pills. Anesthesia \&t Analgesia 2017;125:2105-12.

4. Mullard A. Do you want chips with that? Nat Rev Drug Discov 2015;14:735-7.

5. Mullard A. Fda approves first digital pill. Nat Rev Drug Discov 2017;16:818.

6 Cosgrove L, Cristea IA, Shaughnessy AF, et al. Digital aripiprazole or digital evergreening? A systematic review of the evidence and its dissemination in the scientific literature and in the media. BMJ Evid Based Med 2019;24:231-8.

7. Hitchings AW, Baker EH, Khong TK. Making medicines evergreen. BMJ 2012;345:e7941.

8. Kennedy J, Wood EG. Medication costs and adherence of treatment before and after the Affordable care act: 1999-2015. Am J Public Health 2016;106:1804-7.

9. Belluck P. First digital pill approved to worries about biomedical 'big brother.' The New York Times, 2017. Available: https://www.nytimes.com/ 2017/11/13/health/digital-pill-fda.html [Accessed 26 Jul 2019].

10. Food and Drug Administration. Clinical Review: aripiprazole + MIND 1 system (Abilify Mycite). Center for Drug Evaluation and Research Clinical Review(s) 2017.

11. Downing NS, Ross JS, Jackevicius CA. Avoidance of generic competition by Abbott laboratories' fenofibrate Franchise. Arch Intern Med 2012;172:724-30.

12. Schwartz LM, Woloshin S. How the FDA forgot the evidence: the case of donepezil 23 Mg. BMJ 2012;344:e1086.

13. Frias J, Virdi N, Raja P, et al. Effectiveness of digital medicines to improve clinical outcomes in patients with uncontrolled hypertension and type 2 diabetes: prospective, open-label, cluster-randomized pilot clinical trial. $J$ Med Internet Res 2017;19:e246. 